\title{
An Adjusted Inverse Distance Weighted Spatial Interpolation Method
}

\author{
Zhengquan Li, Kuo Wang*, Hao Ma and Yaoxiang Wu \\ Zhejiang Climate Center, Zhejiang Meteorological Bureau, Hangzhou, 310017, China \\ ${ }^{*}$ Corresponding author
}

\begin{abstract}
Considering the shortcomings of IDW interpolation, this study improved the IDW algorithm and proposed a new spatial interpolation method that is called Adjusted Inverse Distance Weighted (AIDW). The AIDW is capable of taking into account the comprehensive influence of relative distance and position of sample points on the interpolated point, by adding a coefficient (K) to IDW formula. The coefficient (K) is used to adjust the distance weight of sample point according to its shielded effect in sample point positions. Theoretical analysis and case study indicates that the AIDW algorithm could diminish the IDW interpolation error of nonuniform distribution of sample points, consequently the AIDW interpolating is more reasonable, compared with the IDW interpolating. On the other hand, the contour plotting of the AIDW interpolation can effectively avoid the implausible isolated circles and concentric circles that originated from the defect of the IDW interpolation. The contour derived from the AIDW interpolated surface is more similar to the professional manual identification than that from the IDW interpolated surface.
\end{abstract}

Keywords-AIDW; spatial interpolation; contour plotting; IDW

\section{INTRODUCTION}

A basic issue in spatial analysis is interpolating a continuous surface from point samples. Numerous spatial interpolation methods have already been developed for supporting transformation from point data to continuous surface map [1-5]. Those methods can be divided into geographical statistics, non-geographical statistics and hybrid approach. Natural neighbor, splines and trend surface polynomial regression, radial basic function (RBF), triangular irregular network (TIN) and inverse distance weighted (IDW), which are all classified as non-geographical statistics [1,6]. Various forms of Kriging methods can be classified as geographical statistics [7-8]. Hybrid interpolation approach includes regression Kriging, linear mixed model, lapse rate combined with Kriging, and so on [9-12].

Probably one of the most famous interpolation methods is IDW, which is a simple and intuitive deterministic interpolation. IDW is easy to be implemented and available in almost any GIS software, so it is applied frequently in various disciplines [1]. The well-known advantage of IDW is that keeping the measured value at sample location $[5,13]$. But IDW has a shortcoming that it always does not work well on conditions when the sample points are clustering [14-15]. Moreover, many studies have reported that IDW interpolation is inclined to emerge "bull's eye" patterns in contour plotting [16-18]. Some suggestions for enhancing IDW method have been proposed as well. For improving interpolation accuracy, the method of gradient plus inverse distance squared (GIDS) was put forward as a modification for IDW method [19-20]. On the other hand, an adaptive inverse-distance weight that can vary p value of IDW according to sample points clustering pattern was also proposed in some studies [14,21]. However, the published IDW methods only weigh the influence of sample point distance on the estimated point, neglecting the effect of sample point position on the estimated point. Therefore, the interpolating reasonableness of IDW still faces suspicion, especially on the condition that sample points do not evenly distribute surrounding the estimated point.

Considering the comprehensive influence of distance and position of sample point on interpolated point, this study brings out an improved method of IDW interpolation that is named as Adjusted Inverse Distance Weighting (AIDW). In this paper, firstly introduces the application limit of IDW interpolating method. Next, the assumption and expressions of AIDW is given and the advantage of AIDW is described by theoretical analysis. And then, the AIDW performance is analyzed by conducted a case study. Lastly, the study gives a brief conclusion of AIDW application.

\section{ADJUSTED INVERSE DistANCE WEIGHTED}

\section{A. Shortcoming of inverse distance weighted}

IDW interpolation accords to the First Law of Geography "everything is related to everything else, but near things are more related than distant things." [13,22]. The IDW formula is given as (1).

$$
Z=\sum_{i=1}^{n} \frac{1}{\left(d_{i}\right)^{p}} Z_{i} / \sum_{i=1}^{n} \frac{1}{\left(d_{i}\right)^{p}}
$$

Where $\mathrm{Z}$ is the estimated value for prediction point, $\mathrm{Z}_{i}$ is the measured value for sample point, $d_{i}$ is the Euclidean distance between sample point and prediction point, $p$ is a power parameter, and $n$ represents the number of sample points. A main factor affecting IDW interpolation result is the $p$ value. When the $p$ value increases, the smoothness of IDW output surface increases [23].

It is well known that the performance of IDW interpolation is better when sample points distribute regularly, but it faces various suspicions if sample points are clustering, because high clustering of sample points will reduce the accuracy of IDW interpolation [24-26]. Moreover, IDW interpolating surface is sensitive to the presence of outliers, and it suffers from 
discontinuities at sample points resulting in peaks or troughs, especially where sample points are sparse. As a result, the contour map derived from IDW interpolated surface usually shows many isolated circles and concentric circles (commonly called as "bull's eye"), which affects the aesthetic property of contour map [17-18]. To avoid the shortcomings of IDW interpolation, this paper improves IDW algorithm and puts forward an AIDW interpolation method.

\section{B. Assumption of AIDW}

AIDW is similar to IDW abiding by the First Law of Geography. A key difference between AIDW and IDW is adding a coefficient $(K)$ in IDW formula to adjust the distance weight of sample point. The coefficient $(K)$ is based on an assumption that sample point which is close to prediction location has shielding influence on those sample points which are farther away to prediction location, and the shielding influence can reduce the distance weight of shielded sample point. The shielding influence appears when $\alpha<360^{\circ} / n$, and disappears when $\alpha \geq 360^{\circ} / n$. $\alpha$ is an intersection angle for two lines which connect prediction point and two sample points (Figure I), and $n$ is the number of sample points used for the prediction. Figure Ia shows that all sample points distribute evenly surrounding prediction point (black). The intersection angle $(\alpha)$ of random two sample points is greater than or equal to 72 degree $\left(\alpha \geq 360^{\circ} / 5\right)$. AIDW assumes that these sample points have no shielding or shielded influence. In Figure Ic, sample points are high clustering and the maximum intersection angle $(\alpha)$ is lower than 72 degree. On this condition, AIDW assumes that $Z 1$ have shielding influence on $Z_{2}, Z_{3}, Z_{4}$ and $Z_{5}, Z_{2}$ influences $Z_{3}, Z_{4}$ and $Z_{5}, Z_{3}$ influences $Z_{4}$ and $Z_{5}$, and $Z_{4}$ influences $Z_{5}$. In most cases, sample points distributing is more analogous to Figure $\mathrm{Ib}$, neither like Figure Ia nor Figure Ic. The intersection angle $(\alpha)$ of $Z_{1}$ and $Z_{3}$ is equal to 72 degree in Figure Ib, so AIDW assumes that $Z_{1}$ and $Z_{3}$ have no shielding influence on other sample points, but $Z_{2}$ influences $Z_{4}$ and $\mathrm{Z}_{5}$, moreover $\mathrm{Z}_{4}$ influences $\mathrm{Z}_{5}$.

\section{Expressions of AIDW algorithm}

Combining the IDW formula, AIDW algorithm can be expressed as (2).

$$
Z=\sum_{i=1}^{n} \frac{k_{i}}{\left(d_{i}\right)^{p}} Z_{i} / \sum_{i=1}^{n} \frac{k_{i}}{\left(d_{i}\right)^{p}}
$$

Where $\mathrm{Z}, \mathrm{Z}_{i}, d_{i}$ and $n$ is the same as that of the (1), $i$ is a sequence number of sample point which is sorted by the $d_{i}$ value (from low to high), and $k_{i}$ is an adjusted coefficient of distance weight which represents the comprehensive shielded influence of $i$ sample point. The $k_{i}$ is calculated by (3), whose value ranges from zero to 1 .

$$
k_{i}=\left\{\begin{array}{cc}
1 & i=1 \\
\prod_{j=1}^{j=i-1} \sin ^{p} \theta_{i j} & i=2,3,4 \cdots n
\end{array}\right.
$$

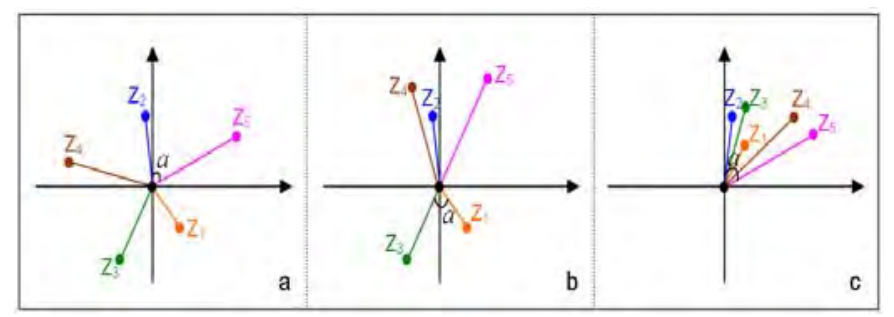

FIGURE I. EXAMPLES OF SAMPLE POINTS SCATTERING IN INTERPOLATING NEIGHBORHOOD (FIVE POINTS)

where $\sin ^{p} \theta_{i j}$ is used to weigh the shielded influence of $i$ sample point by $j$ sample point $(1 \leq j \leq i-1)$. In (3), $k_{i}$ is expressed as a multiplicative model of $\sin ^{p} \theta_{i j}$. As a result, the more sample points shield $i$ point, the lower $k_{i}$ value is, and the interpolating weight of $i$ point decreases much more. Due to the closest point to prediction location is not shielded by any other sample point, so $k_{1}$ is set to 1 .

The $\sin ^{p} \theta_{i j}$ can be calculated by (4).

$$
\sin ^{p} \theta_{i j}=\left\{\begin{array}{cc}
1 & \alpha_{i j} \geq 360^{\circ} / n \\
\left(1-\cos ^{2} \theta_{i j}\right)^{\frac{p}{2}} & \alpha_{i j}<360^{\circ} / n
\end{array}\right.
$$

where $\theta_{i j}$ is the intersection angle formed by the line that connects $i$ and $j$ sample points and the line that passes through prediction point and the midpoint of line $1 . \alpha_{i j}$ is the intersection angle for two lines that connect prediction point, $i$ and $j$ sample points separately. $\theta_{i j}$ and $\alpha_{i j}$ are illustrated in Figure II.

Integrating (2) with (3), AIDW algorithm can also be expressed as the following.

$$
Z=\frac{\frac{Z_{1}}{d_{1}^{p}}+\frac{Z_{2}}{d_{2}^{p}} \sin ^{p} \theta_{21}+\frac{Z_{3}}{d_{3}^{p}} \sin ^{p} \theta_{31} \sin ^{p} \theta_{32}+\cdots+\frac{Z_{n}}{d_{n}^{p}} \sin ^{p} \theta_{n 1} \sin ^{p} \theta_{n 2} \sin ^{p} \theta_{n 3} \cdots \sin ^{p} \theta_{n, n-1}}{\frac{1}{d_{1}^{p}}+\frac{1}{d_{2}^{p}} \sin ^{p} \theta_{21}+\frac{1}{d_{3}^{p}} \sin ^{p} \theta_{31} \sin ^{p} \theta_{32}+\cdots+\frac{1}{d_{n}^{p}} \sin ^{p} \theta_{n 1} \sin ^{p} \theta_{n 2} \sin ^{p} \theta_{n 3} \cdots \sin ^{p} \theta_{n, n-1}}
$$

\section{Capability of weighing shielded influence}

In course of $\mathrm{Z}_{i}$ moving to prediction location $\left(\mathrm{Z}_{o}\right)$ with a constant angle (Figure IIa), $\theta$ and $\sin ^{p} \theta$ values are increasing which indicates that the shielding influence of $Z_{j}$ on $Z_{i}$ is gradually reducing. When $Z_{i}$ moves to the point whose distance between $Z_{i}$ and $Z_{o}$ is equal to the distance between $Z_{j}$ and $Z_{o}$, the three points $\left(Z_{o}, Z_{j}\right.$ and $\left.Z_{i}\right)$ form an isosceles triangle. On this condition, $\theta$ is 90 degree, and $\sin ^{p} \theta$ value is $l$, which means that $\mathrm{Z}_{j}$ has no shielding influence on $\mathrm{Z}_{i}$ anymore.

In process of $Z_{i}$ moving to right behind $Z_{j}$ with a constant distance between $Z_{i}$ and $Z_{o}$ (Figure IIb), $\theta$ and $\sin ^{p} \theta$ values are decreasing, which indicates the shielding influence of $Z_{j}$ on $Z_{i}$ is gradually enhancing. If $\theta$ and $\sin ^{p} \theta$ value is 0 which means $Z_{j}$ completely shields $Z_{i}$. That is to say, $Z_{i}$ is useless for estimating $\mathrm{Z}_{o}$. 
Regarding the moving places of $\mathrm{Z}_{i}$ as the locations of other sample points, Figure II illustrates the variation of $\theta$ and $\sin ^{p} \theta$ in two extreme cases of sample point distribution. One case is that the distances between two sample points and prediction point are tending to be equal (Figure IIa), $\theta \rightarrow 90^{\circ}, \quad \sin ^{p} \theta \rightarrow 1$. The other case is that one sample point is tending to right behind the other points (Figure IIb), $\theta \rightarrow 0^{\circ}$, $\sin ^{p} \theta \rightarrow 0$.

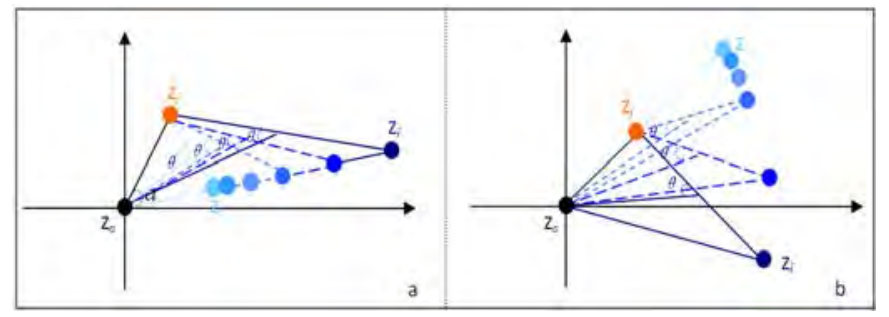

FIGURE II. EXAMPLES OF $\sin ^{p} \theta$ VALUE CHANGING WITH SAMPLE POINT MOVING

The two extreme cases can cover all position relationship of random two sample points; therefore it is reasonable using $\sin ^{p} \theta$ to weigh the shielding or shielded influence of sample points.

\section{E. Characteristic of AIDW interpolation}

By adding the adjusted coefficient $(K)$ in IDW formula, AIDW can consider the comprehensive influence of distance and position of sample point. When the distribution of sample points is even (Figure Ia), or the distances of all points to prediction point are same, the interpolating result of AIDW is the same as that of IDW interpolating, so IDW can be regarded as a special case of AIDW. When sample points are clustering in a section such as Figure III, AIDW can make sample points that are used for interpolating tend to even distributing. On the other hand, AIDW is capable of reducing the probability of "bull's eye" emerging in contour plotting (Figure VI).

Figure IIIa shows sample points which are high clustering in right-upper section of prediction point (black point), the result of IDW interpolating cannot be guaranteed due to contravening the principle of geo-statistical analysis that sample points are even distribute. However, considering the shielding or shielded influence of sample points, AIDW interpolating will reduce the distance weights of $Z_{6}, Z_{7}$ and $Z_{8}$ ( $\mathrm{Z}_{6}$ shielded by $\mathrm{Z}_{1}, \mathrm{Z}_{7}$ shielded by $\mathrm{Z}_{3}$ and $\mathrm{Z}_{4}$, and $\mathrm{Z}_{8}$ shielded by $Z_{3}$ ), and make the distance weights of $Z_{10}, Z_{11}$ and $Z_{12}$ to be zero $\left(Z_{10}, Z_{11}\right.$ and $Z_{12}$ is completely shielded by $Z_{4}, Z_{7}$ and $Z_{8}$, respectively), as shown in Figure IIIb. Consequently, the value of prediction location is estimated by (6). Because the AIDW interpolating of prediction point mainly considers purple points and partly considers green points (Figure IIIb), AIDW has made sample points tend to distribute evenly.

$Z=\frac{\frac{Z_{1}}{d_{1}^{p}}+\frac{Z_{2}}{d_{2}^{p}}+\frac{Z_{3}}{d_{3}^{p}}+\frac{Z_{4}}{d_{4}^{p}}+\frac{Z_{5}}{d_{5}^{p}}+\frac{Z_{6} \sin ^{p} \theta_{61}}{d_{6}^{p}}+\frac{Z_{7} \sin ^{p} \theta_{73} \sin ^{p} \theta_{74}}{d_{7}^{p}}+\frac{Z_{8} \sin ^{p} \theta_{83}}{d_{8}^{p}}+\frac{Z_{9}}{d_{9}^{p}}}{\frac{1}{d_{1}^{p}}+\frac{1}{d_{2}^{p}}+\frac{1}{d_{3}^{p}}+\frac{1}{d_{4}^{p}}+\frac{1}{d_{5}^{p}}+\frac{\sin ^{p} \theta_{61}}{d_{6}^{p}}+\frac{\sin ^{p} \theta_{73} \sin ^{p} \theta_{74}}{d_{7}^{p}}+\frac{\sin ^{p} \theta_{83}}{d_{8}^{p}}+\frac{1}{d_{9}^{p}}}$
In order to concisely compare contour plotting of IDW and AIDW interpolation, a simply example is given in Figure VI. The distance of purple point to prediction point (black point) is same and its value is 1 . Tawny points lie right behind purple points, and their distances to prediction point are also same (whose value is 2). The value of prediction point estimated by IDW (setting $p=1$ ) is 50 , while the value estimated by AIDW (setting $p=1$ ) is 20 , because tawny points are shielded completely by purple points which causes the weights of tawny points reducing to zero. From this simply example, it can be seen clearly that the interpolating result of AIDW is more reasonable than IDW.

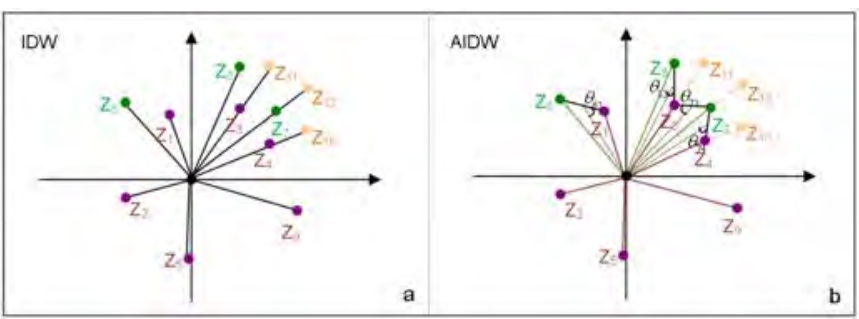

FIGURE III. DIFFERENT TREATING MODE OF SAMPLE POINTS IN IDW AND AIDW INTERPOLATION METHODS

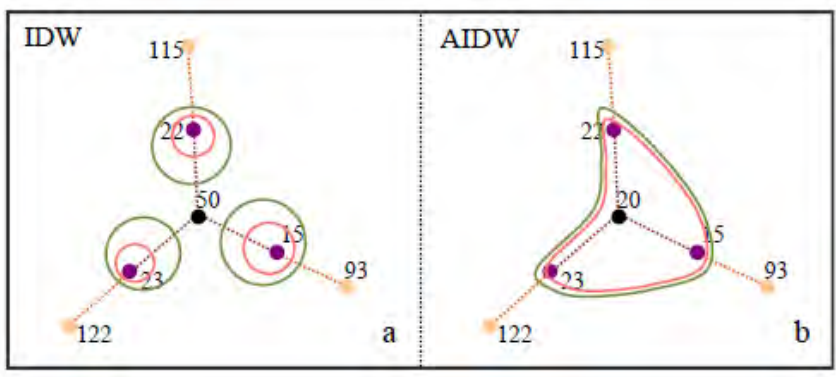

FIGURE IV. CONTOUR PLOTTING EXAMPLE OF IDW AND AIDW INTERPOLATION METHODS (THE VALUE OF RED AND GREEN CONTOURS IS 30 AND 40, RESPECTIVELY)

As shown in Figure VIa, since the low values of purple points surround the higher value of prediction point estimated by the IDW, the chance of the concentric circles pattern emerging would increase in contour plotting. However, the value of prediction point estimated by the AIDW is close to the values of purple points, therefore prediction point and purple points could be classified into a same zone, avoiding the appearance of the concentric circles pattern (Figure VI b). As for a case study of AIDW and IDW in contour plotting, it is given in Section III.

\section{CASE STUdy OF AIDW AND IDW INTERPOLATION}

Based on annual precipitation data from 71 weather stations in Zhejiang province, this study had interpolated precipitation surface by individually applying IDW and AIDW method. In the interpolating process, setting the power parameter (p) was 2 , interpolating neighborhood included 5 weather stations, and the spatial resolution of precipitation surface was $1 \mathrm{~km} \times \mathrm{l} \mathrm{km}$. IDW interpolating and its contour plotting were conducted by ArcMap9.3 software, and AIDW was conducted by the programs mentioned above. The errors of the IDW and AIDW interpolating for annual precipitation are listed in Table I. 
Compared to IDW interpolation, the result of AIDW interpolation is more reliable. The errors of AIDW interpolation are all lower than that of IDW interpolation, especially in the mean error and the root mean square error.

For the contour plotting, though the surface and contours resulted from AIDW method have a broadly similar pattern with that from IDW method, there are significant differences in local areas marked by the rectangular (Figure V). The "bull's eye" (in the red rectangular) and isolated circle (in the black rectangular) in Figure V a are disappeared in Figure Vb.

TABLE I ERRORS ANALYSIS OF CROSS VALIDATION OF THE IDW AND AIDW INTERPOLATION

\begin{tabular}{ccccc}
\hline $\begin{array}{c}\text { Interpolation } \\
\text { Method }\end{array}$ & $\begin{array}{c}\text { Mean } \\
\text { Error } \\
/ \mathbf{m m}\end{array}$ & $\begin{array}{c}\text { Mean } \\
\text { Absolute } \\
\text { Error } \\
/ \mathbf{m m}\end{array}$ & $\begin{array}{c}\text { Mean } \\
\text { Relative } \\
\text { Error } \\
/ \%\end{array}$ & $\begin{array}{c}\text { Root Mean } \\
\text { Square } \\
\text { Error } \\
/ \mathbf{m m}\end{array}$ \\
\hline IDW & 8.2 & 95.9 & $7.1 \%$ & 179.8 \\
AIDW & 3.9 & 87.3 & $6.5 \%$ & 136.3 \\
\hline
\end{tabular}

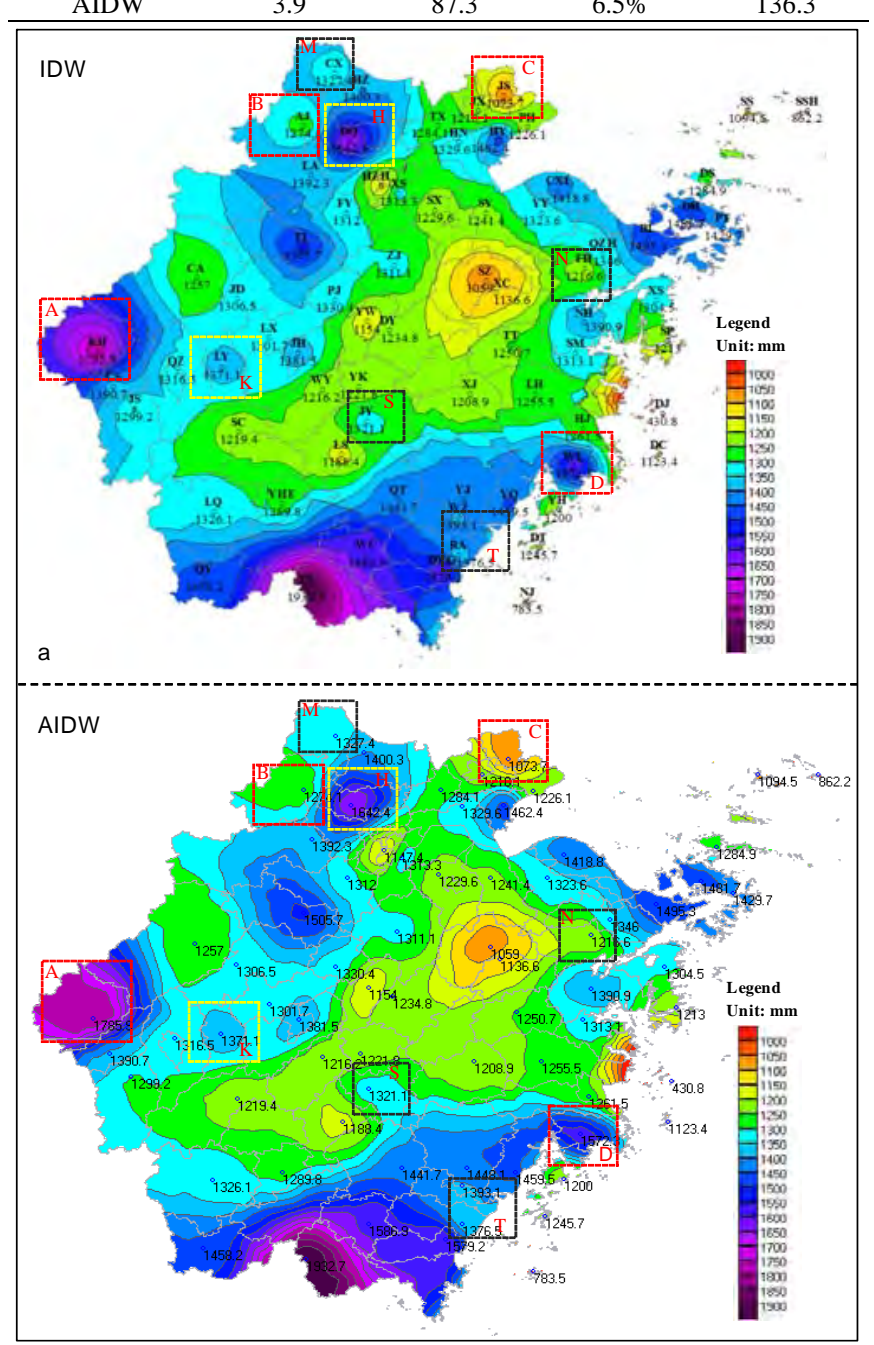

FIGURE V. COMPARISON OF CONTOUR PLOTTING OF IDW AND AIDW INTERPOLATION METHODS

In the area marked by letter $A$, the annual precipitation of $\mathrm{KH}$ station is much higher than that of its surrounding stations (CS, JS, QZ and CA station). According to IDW algorithm, the weight of $\mathrm{KH}$ station decreases with prediction location being far away from $\mathrm{KH}$ station, meanwhile the sum weight of its surrounding station is relatively increasing. As a result, the further away from $\mathrm{KH}$ station, the smaller an estimated value of prediction location is, which results in a "bull's eye" pattern surrounding $\mathrm{KH}$ station (Figure $\mathrm{Va}$ ). In the interpolating process of AIDW method (especially for the interpolations of the left area of $\mathrm{KH}$ station), $\mathrm{KH}$ station has shielding influences on CS, JS, QZ and CA station, and the four stations have also shielding or shielded influences on each other. Consequently, the weights of surrounding stations are reduced relatively and the estimating of prediction location is mainly controlled by $\mathrm{KH}$ station. Since little difference among the estimated values in the left area of $\mathrm{KH}$ station, these estimated values are classified into a same zone which avoids "bull's eye" emerging in contour plotting (Figure $\mathrm{Vb}$ ). In those areas marked using letter $\mathrm{B}, \mathrm{C}$ or $\mathrm{D}$, the interpretation about the differences between IDW and AIDW interpolating is analogous to that in the area $\mathrm{A}$.

Besides reducing the probability of "bull's eye" emerging, AIDW has a capability to decrease the chance of isolated circle appearing. In the area marked by letter $\mathrm{M}$, there is an isolated circle surrounding $\mathrm{CX}$ station (Figure $\mathrm{Va}$ ). Owning to $\mathrm{HZ}$ and DQ stations existing, the value estimated by IDW method becomes greater while the prediction location is further away from CX station, which results in the appearance of isolated circle. In the interpolating process of AIDW method (especially for the interpolations of the left area of CX station), HZ station is subjected to the shielding influence of CX station, and DQ station suffers the corporative shielding influences of $\mathrm{CX}, \mathrm{HZ}$ and AJ station. Therefore, the contribution of DQ station to the estimated value is greatly depressed, and HZ station is partly decreased too. As a result, the interpretation of the left area of CX station is mainly dominated by CX and AJ stations, which results in the similar interpolated values, so the isolated circle does not appear in Figure $\mathrm{Vb}$. In those areas marked using letter $\mathrm{N}, \mathrm{S}$ or $\mathrm{T}$, the interpretation for appearing or disappearing of the isolated circle is analogous to that of the area $\mathrm{M}$.

It is worth noting that AIDW has not eliminated the "bull eye" at DQ station and isolated circle at LY station which marked by the yellow rectangular frame (Figure V), because the four stations surrounding DQ or LY station distribute evenly (one in each quadrant area). It demonstrated that AIDW is similar to IDW when the distribution of sample points is even.

Generally speaking, the precipitation surface interpolated by AIDW method is more reasonable than IDW method, especially for the interpolations of the surface frontier areas. Moreover, AIDW method can reduce the number of implausible isolated circle and concentric circles objectively, and its contour plotting is smoother comparing with IDW method.

\section{Conclusion And Discussion}

Though IDW method is commonly used in spatial analysis, it has the shortcomings of neglecting sample points clustering, and inclining to emerge isolated circle and concentric circles in its contour plotting. For the sake of overcoming above problems, this study had improved IDW algorithm and put 
forward a new method called AIDW. According to the intersection angle $(\alpha)$, AIDW decides whether or not to have shielding influences among sample points, and weighs the shielded contribution of the point shielded by other points using the coefficient (K). Theoretical analysis and the case study indicated that AIDW method could acquire more reasonable interpolations than IDW method. Moreover, the contour plotting of AIDW can effectively avoid the implausible isolated circles and concentric circles which often exist in IDW interpolating; its contour plotting has more rationality and aesthetic.

Due to involving the calculation of $\alpha$ and $\sin ^{p} \theta$, the computing efficiency of AIDW method is slight lower than that of IDW method. In the above case study, the computational time of either AIDW or IDW was less than $1 \mathrm{~s}$. But the running time of AIDW method is increasing more than IDW method, while the interpolating resolution is higher and interpolating neighborhood including more sample points. When interpolating resolution was $500 \mathrm{~m} \times 500 \mathrm{~m}$ and the interpolating neighborhood included 15 weather stations, AIDW method had cost $11 \mathrm{~s}$ to conduct interpolating precipitation surface of Zhejiang province, and IDW method consumed $7 \mathrm{~s}$ to do the same thing. However, it is worth getting the interpolating reasonability regardless of the slight more running time.

\section{ACKNOWLEDGMENT}

This study was supported by the Public Welfare Technology Research Projects of Zhejiang Province (2015C33055), Natural Science Foundation of China (41605049), and Fund for Meteorological Science and Technology of Zhejiang Province, China (2017ZD13, 2017QN04).

\section{REFERENCES}

[1] J. Li. and A. D. Heap. "A review of spatial interpolation methods for environmental scientists,” Canberra: Geoscience Australia, 2008.

[2] M. Delbari, P. Afrasiab, S. Jahani. "Spatial interpolation of monthly and annual rainfall in northeast of Iran,” Meteorol Atmos Phys, 2013, 122(12), 103-113.

[3] S. Wang, G. H. Huang, Q. G. Lin, et al. "Comparison of interpolation methods for estimating spatial distribution of precipitation in Ontario, Canada,” Int J Climatol, 2014, 34(14), 3745-3751.

[4] P. D. A. Borges, J. Franke, H. Weiss, and C. Bernhofer. "Comparison of spatial interpolation methods for the estimation of precipitation distribution in distrito federal, Brazil," Theor Appl Climatol, 2016, 123(1-2), 335-348.

[5] T. Chen, L. L. Ren, and F. Yuan. "Comparison of spatial interpolation schemes for rainfall data and application in hydrological modeling," Water, 2017, 9(5), 342-342.

[6] F. W. Chen and C. W. Liu. "Estimation of the spatial rainfall distribution using inverse distance weighting (IDW) in the middle of Taiwan," Paddy Water Environ, 2012, 10, 209-222.

[7] H. Apaydin, F. K. Sonmez, and Y. E. Yildirim. "Spatial interpolation techniques for climate data in the gap region in Turkey," Clim Res, 2004, 28, 31-40.
[8] H. C. Yeh, Y. C. Chen, C. Wei, and R. H. Chen. "Entropy and kriging approach to rainfall network design,” Paddy Water Environ, 2011, 93, 343-355.

[9] X. Li, G. Cheng, and L. Lu. "Spatial analysis of air temperature in the Qinghai-Tibet Plateau,” Arct Antarct Alp Res, 2005, 37, 246-252.

[10] W. Z. Shi and Y. Tian. "A hybrid interpolation method for the refinement of a regular grid digital elevation model,” Int J Geogr Inf Sci, 2006, 20, 53-67.

[11] E. Ozelkan, S. Bagis, E. C. Ozelkan, et al. "Spatial interpolation of climatic variables using land surface temperature and modified inverse distance weighting,” Int J Remote Sens, 2015, 36(4), 1000-1025.

[12] Y. H. Wang, H. B. Yang, D. W. Yang, et al. "Spatial interpolation of daily precipitation in a high mountainous watershed based on gauge observations and a regional climate model Simulation,” J Hydrometeorol, 2016, 18(3), 845-862.

[13] K. Johnston, J. M. V. Hoef, K. Krivoruchko, and N. Lucas. "Using ArcGIS geostatistical analyst,” New York: Environmental systems research institute, Inc. 2001.

[14] G. Y. Lu and D. W. Wong. "An adaptive inverse-distance weighting spatial interpolation technique,” Comput Geosci, 2008, 34, 1044-1055.

[15] M. Azpurua and K. D. Ramos. "Comparison of spatial interpolation methods for estimation of average electromagnetic field magnitude," Prog Electromagn Res M, 2010, 14, 135-145.

[16] C. A. Gotway, R. B. Ferguson, G. W. Hergert, and T. A. Peterson. "Comparison of kriging and inverse distance methods for mapping soil parameters,” Soil Sci Soc Am J, 1996, 60, 1237-1247.

[17] Z. M. Feng, Y. Z. Yang, X. Q. Ding, and Z. H. Lin. "Optimization of the spatial interpolation methods for climate resources,” Geogr Res, 2004, 23, 357-364.

[18] D. Nusert and S. Dug. “Applying the inverse distance weighting and kriging methods of the spatial interpolation on the mapping the annual precipitation in Bosnis and Rzegovina,” In: R. Seppelt, A. A. Voinov, S. Lange, D. Bankamp. International Congress on Environmental Modeling and Software. Leipzig: iEMSs. 2012.

[19] J. Hooyberghs, C. Mensink, G. Dumont, and F. Fierens. "Spatial interpolation of ambient ozone concentrations from sparse monitoring points in Belgium,” J Environ Monitor, 2006, 8, 1129-1135.

[20] N. S. Golkhatmi, S. H. Sanaeinejad, B. Ghahraman, et al. "Extended modified inverse distance method for interpolation rainfall," Inter J Eng Inv, 2012, 1, 57-65.

[21] G. Y. Lu, L. S. Chiu, and D. W. Wong. "Vulnerability assessment of rainfall-induced debris flows in Taiwan,” Nat Hazards, 2007, 43, 223244.

[22] W. R. Tobler. "A computer movie simulating urban growth in the Detroit region,” Econ Geogr, 1970, 46, 234-240.

[23] R. B. Husar, and S. R. Falke. "Uncertainty in the spatial interpolation of PMlO monitoring data in Southern California,” [online], 1996, http://capita.wustl.edu/CAPITAJcapitareports/cainterp/cainterp.doc.

[24] D. Zimmerman, C. Pavlik, A. Ruggles, and M. P. Armstrong. “An experimental comparison of ordinary and universal kriging and inverse distance weighting,” Math Geol, 1999, 31, 375-390.

[25] K. Stahl, R. D. Moore, J. A. Floyer, M. G. Asplin, and I. G. Mckendry. "Comparison of approaches for spatial interpolation of daily air temperature in a large region with complex topography and highly variable station density,” Agr Forest Meteorol, 2006, 139, 224 - 236.

[26] K. Wang, H. Ma, J. Li, B. H. Gu, and H. Wu. "Assessment of the POEM2 model for simulating tropical intraseasonal oscillation,” J Trop Meteorol, 2018, 24(3): 323-333. 